\title{
AUTOMORPHISMS OF THE UNIMODULAR GROUP
}

BY

\author{
L. K. HUA AND I. REINER
}

Notation. Let $\mathfrak{M}_{n}$ denote the group of $n \times n$ integral matrices of determinant \pm 1 (the unimodular group). By $\mathfrak{M}_{n}^{+}$we denote that subset of $\mathfrak{M}_{n}$ where the determinant is $+1 ; \mathfrak{M}_{n}^{-}$is correspondingly defined. Let $I^{(n)}$ (or briefly $I$ ) be the identity matrix in $\mathfrak{M}_{n}$, and let $X^{\prime}$ represent the transpose of $X$. The direct sum of the matrices $A$ and $B$ will be represented by $A \dot{+} B$;

$$
A \stackrel{8}{=} B
$$

will mean that $A$ is similar to $B$. In this paper, we shall find explicitly the generators of the group $\mathfrak{A}_{n}$ of all automorphisms of $\mathfrak{M}_{n}$.

1. The commutator subgroup of $\mathfrak{M}_{n}$. The following result is useful, and is of independent interest.

THEOREM 1. Let $\mathfrak{R}_{n}$ be the commutator subgroup of $\mathfrak{M}_{n}$. Then trivially $\Re_{n} \subset \mathfrak{M}_{n}^{+}$. For $n=2, \Re_{n}$ is of index 2 in $\mathfrak{M}_{n}^{+}$, while for $n>2, \Re_{n}=\mathfrak{M}_{n}^{+}$.

Proof. Consider first the case where $n=2$. Define

$$
S=\left(\begin{array}{rr}
0 & 1 \\
-1 & 0
\end{array}\right), \quad T=\left(\begin{array}{ll}
1 & 1 \\
0 & 1
\end{array}\right)
$$

It is well known that $S$ and $T$ generate $\mathfrak{M}_{2}^{+}$. An element $X$ of $\mathfrak{M}_{2}^{+}$is called even if, when $X$ is expressed as a product of powers of $S$ and $T$, the sum of the exponents is even; otherwise, $X$ is called odd. Since all relations satisfied by $S$ and $T$ are consequences of

$$
S^{2}=-I, \quad(S T)^{3}=I,
$$

it follows that the parity of $X \in \mathfrak{M}_{2}^{+}$depends only on $X$, and not on the manner in which $X$ is expressed as a product of powers of $S$ and $T$. Let $\&$ be the subgroup of $\mathfrak{M}_{2}^{+}$consisting of all even elements; then clearly $\&$ is of index 2 in $\mathfrak{M}_{2}^{+}$. It suffices to prove that $\mathbb{E}=\mathfrak{R}_{2}$.

We prove first that $\Re_{2} \subset \mathbb{E}$. Since the commutator subgroup of a group is always generated by squares, it suffices to show that $A \in \mathfrak{M}_{2}$ implies $A^{2} \in \mathbb{E}$. For $A \in \mathfrak{M}_{2}^{+}$, this is clear. If $A \in \mathfrak{M}_{2}^{-}$, set $A=X J=J Y$, where

$$
J=\left(\begin{array}{rr}
1 & 0 \\
0 & -1
\end{array}\right)
$$

Presented to the Society, December 29, 1950; received by the editors January 8, 1951. 
and $X$ and $Y \in \mathfrak{M}_{2}^{+}$. Then $A^{2}=X Y=X J^{-1} X J$. Hence we need only prove that if $X \in \mathfrak{M}_{2}^{+}, X$ and $J^{-1} X J$ are of the same parity. This is easily verified for $X=S$ or $T$; since $S$ and $T$ generate $\mathfrak{M}_{2}^{+}$, and $J^{-1} X_{1} X_{2} J=J^{-1} X_{1} J \cdot J^{-1} X_{2} J$, the result follows.

On the other hand we can show that $\& \subset \Re_{2}$. For, $\&$ is generated by $T^{2}$ and $S T$, since $T S=\left(S T \cdot T^{-2}\right)^{2}$. However, $T^{2}=T J T^{-1} J^{-1} \in \Re_{2}$, and therefore also $\left(T^{\prime}\right)^{-2} \in \Re_{2}$. Furthermore, $S T=T S T^{-1} S^{-1}\left(T^{\prime}\right)^{-2} T^{2} \in \Re_{2}$. This completes the proof for $n=2$.

Suppose now that $n>2$, and define

$$
\begin{aligned}
& R=\left(\begin{array}{ccc}
0 \cdots & \cdots & (-1)^{n-1} \\
1 \cdots & 0 & 0 \\
\cdots & . & \cdot \\
0 & \cdots & 1
\end{array}\right) \in \mathfrak{M}_{n}^{+}, \quad S=\left(\begin{array}{rr}
0 & 1 \\
-1 & 0
\end{array}\right)+I^{(n-2)} \\
& T=\left(\begin{array}{ll}
1 & 1 \\
0 & 1
\end{array}\right)+I^{(n-2)} .
\end{aligned}
$$

(The symbols $S$ and $T$ defined here are the analogues in $\mathfrak{M}_{n}^{+}$of those defined by (1). It will be clear from the context which are meant.) For $n>2$ we have $(1)$

$$
T^{\prime}=\left[R^{-1}(T R)^{-(n-2)} R(T R)^{n-2}\right](T R)^{-1}\left[R(T R)^{-(n-2)} R^{-1}(T R)^{n-2}\right](T R) \in \Re_{n} .
$$

Further $S=T S T^{-1} S^{-1}\left(T^{\prime}\right)^{-2} T \in \Re_{n}$. Finally, for odd $n$ there exists a permutation matrix $P$ such that $R^{2}=P^{-1} R P$, whence $R=R^{-1} P^{-1} R P \in \Re_{n}$. For even $n$, $R$ represents the monomial transformation

$$
\left(\begin{array}{llr}
x_{1} & x_{2} \cdots x_{n-1} & x_{n} \\
x_{2} & x_{3} \cdots x_{n} & -x_{1}
\end{array}\right)
$$

which is a product of

$$
\begin{aligned}
& \left(\begin{array}{rrrr}
x_{1} & x_{2} & x_{3} \cdots x_{n-1} & x_{n} \\
x_{2}-x_{1} & x_{3} \cdots x_{n-1} & x_{n}
\end{array}\right), \quad\left(\begin{array}{cccc}
x_{1} & x_{2} & x_{3} & x_{4} \cdots x_{n} \\
-x_{3} & x_{2} & x_{1} & x_{4} \cdots x_{n}
\end{array}\right) . \\
& \left(\begin{array}{rrrr}
x_{1} & x_{2} & x_{3} & x_{4} \cdots x_{n} \\
x_{4} & x_{2} & x_{3}-x_{1} \cdots & x_{n}
\end{array}\right), \cdots,\left(\begin{array}{ccc}
x_{1} & x_{2} \cdots x_{n-1} & x_{n} \\
x_{n} & x_{2} \cdots x_{n-1} & -x_{1}
\end{array}\right) .
\end{aligned}
$$

each factor of which is similar to $S$ (and hence is in $\Re_{n}$ ). Since $T$ and $R$ generate $\mathfrak{M}_{n}^{+}$, the theorem is proved.

COROLLARY 1. In any automorphism of $\mathfrak{M}_{n}$, always $\mathfrak{M}_{n}^{+} \rightarrow \mathfrak{M}_{n}^{+}$.

Proof. For $n>2$ this is an immediate corollary, since the commutator subgroup goes into itself in any automorphism. For $n=2$, let $S \rightarrow S_{1}$ and

(1) L. K. Hua and I. Reiner, Trans. Amer. Math. Soc. vol. 65 (1949) p. 423. 
$T \rightarrow T_{1}$. Then $S T \in \Re_{2}$ implies $S_{1} T_{1} \in \Re_{2}$, so det $\left(S_{1} T_{1}\right)=1$. Further, $S^{2}=-I$ implies $S_{1}^{2}=-I$, so det $S_{1}=1$, since the minimum function of $S_{1}$ is $x^{2}+1$, and the characteristic function must therefore be a power of $x^{2}+1$. This completes the proof when $n=2$.

2. Automorphisms of $\mathfrak{M}_{2}^{+}$. We wish to determine the automorphisms of $\mathfrak{M}_{2}$. Since every automorphism of $\mathfrak{M}_{2}$ takes $\mathfrak{M}_{2}^{+}$into itself, we shall first determine all automorphisms of $\mathfrak{M}_{2}^{+}$. For $X \in \mathfrak{M}_{2}^{+}$, define $\epsilon(X)=+1$ or -1 , according as $X$ is even or odd.

THEOREM 2. Every automorphism of $\mathfrak{M}_{2}^{+}$is of one of the forms

$$
X \in \mathfrak{M}_{2}^{+} \rightarrow A X A^{-1}
$$

$A \in \mathfrak{M}_{2}$

or

$$
X \in \mathfrak{M}_{2}^{+} \rightarrow \epsilon(X) \cdot A X A^{-1},
$$

$A \in \mathfrak{M}_{2}$.

That is, the automorphism group of $\mathfrak{M}_{2}^{+}$is generated by the set of "inner" automorphisms $X \rightarrow A X A^{-1}\left(A \in \mathfrak{M}_{2}\right)$ and the automorphism $X \rightarrow \epsilon(X) \cdot X$.

Proof. Let $\tau$ be an automorphism of $\mathfrak{M}_{2}^{+}$; it certainly leaves $I^{(2)}$ and $-I^{(2)}$ individually unaltered. Let $S$ and $T$ (as given by (1)) be mapped into $S^{r}$ and $T^{r}$. Then $\left(S^{r}\right)^{2}=-I$. Since all second order fixed points are equivalent, there exists a matrix $B \in \mathfrak{M}_{2}$ such that $B S^{\tau} B^{-1}=S$. Instead of $\tau$, consider the automorphism $\tau^{\prime}: X \rightarrow B X^{\tau} B^{-1}$, which leaves $S$ unaltered. Assume hereafter that $\tau$ leaves $S$ invariant. (It is this sort of replacement of $\tau$ by $\tau^{\prime}$ which we shall mean when we refer to some property holding "after a suitable inner automorphism.") Set

$$
T^{\tau}=\left(\begin{array}{ll}
a & b \\
c & d
\end{array}\right)
$$

From $(S T)^{3}=I$ we obtain $\left(S T^{r}\right)^{3}=I$, whence $b-c=1$. Since det $T^{r}=1$, we get

$$
a d=1+b c=c^{2}+c+1>0 .
$$

Set $N=|a+d|$. If $N \geqq 3$, consider the elements generated by $S$ and $T^{r}$ $(\bmod N)$. Since $a+d \equiv 0(\bmod N)$, we find that $\left(T^{\tau}\right)^{2} \equiv I(\bmod N)$. Furthermore $\left(S T^{r}\right)^{3} \equiv I(\bmod N)$; therefore $S$ and $T^{r}$ generate $(\bmod N)$ at most the 12 elements

$$
\pm I, \pm S, \pm T^{\tau}, \pm S T^{\tau}, \pm T^{\tau} S, \pm S T^{\tau} S .
$$

But if $\tau$ is an automorphism, $S$ and $T^{r}$ generate $\mathfrak{M}_{2}^{+}$, which has more than 12 elements $(\bmod N)$ for $N \geqq 3$.

Therefore $N \leqq 2$. Since $a d>0$, either $a=d=1$ or $a=d=-1$, and thence $b=1, c=0$ or $b=0, c=-1$. There are 4 possibilities for $T^{r}$ : 


$$
T^{\tau}= \begin{cases}T_{0}=\left(\begin{array}{ll}
1 & 1 \\
0 & 1
\end{array}\right), & T_{2}=\left(\begin{array}{rr}
-1 & 1 \\
0 & -1
\end{array}\right), \\
T_{1}=\left(\begin{array}{rr}
1 & 0 \\
-1 & 1
\end{array}\right), & T_{3}=\left(\begin{array}{rr}
-1 & 0 \\
-1 & -1
\end{array}\right) .\end{cases}
$$

Since $S$ and $T$ generate $\mathfrak{M}_{2}^{+}$, to determine $\tau$ it is sufficient to specify $S^{\tau}$ and $T^{\tau}$. Thus every automorphism of $\mathfrak{M}_{2}^{+}$is of the form $S \rightarrow B S B^{-1}, T \rightarrow B T_{i} B^{-1}$ (for some $i, i=0,1,2,3$ ), where $B \in \mathfrak{M}_{2}$. If $J$ is given by (2), we have:

$$
T_{0}=T, \quad T_{1}=S T S^{-1}, \quad T_{2}=-J T J^{-1}, \quad T_{3}=-S J T J^{-1} S^{-1},
$$

and also $S=-J S J^{-1}$. The possible automorphisms are:

$$
\begin{array}{lll}
i=0: & S \rightarrow B S B^{-1}, & T \rightarrow B T B^{-1} . \\
i=1: & S \rightarrow B S \cdot S \cdot S^{-1} B^{-1}, & T \rightarrow B S \cdot T \cdot S^{-1} B^{-1} . \\
i=2: & S \rightarrow-B J \cdot S \cdot J^{-1} B^{-1}, & T \rightarrow-B J \cdot T \cdot J^{-1} B^{-1} . \\
i=3: & S \rightarrow-B S J \cdot S \cdot J^{-1} S^{-1} B^{-1}, & T \rightarrow-B S J \cdot T \cdot J^{-1} S^{-1} B^{-1} .
\end{array}
$$

These automorphisms are of two types: for $i=0$ and $1, S \rightarrow A S A^{-1}, T \rightarrow A T A^{-1}$, which imply that $X \in \mathfrak{M}_{2}^{+} \rightarrow A X A^{-1}$; for $i=2$ and $3, S \rightarrow-A S A^{-1}, T \rightarrow-A T A^{-1}$, which imply that $X \in \mathfrak{M}_{2}^{+} \rightarrow \epsilon(X) \cdot A X A^{-1}$. This completes the proof.

3. Automorphisms of $\mathfrak{M}_{n}^{+}$and $\mathfrak{M}_{n}$. We are now faced with the problem of determining the automorphisms of $\mathfrak{M}_{2}$ from those of $\mathfrak{M}_{2}^{+}$. We shall have the same problem for $\mathfrak{M}_{n}$ and $\mathfrak{M}_{n}^{+}$. As we shall see, the passage from $\mathfrak{M}_{n}^{+}$to $\mathfrak{M}_{n}$ is trivial, and most of the difficulty lies in determining the automorphisms of $\mathfrak{M}_{n}^{+}$. In this paper we shall prove the following results:

THEOREM 3. For $n>2$, the group of those automorphisms of $\mathfrak{M}_{n}^{+}$which are induced by automorphisms of $\mathfrak{M}_{n}$ is generated by

(i) the set of all "inner" automorphisms

$$
X \in \mathfrak{M}_{n}^{+} \rightarrow A X A^{-1}
$$

and

(ii) the automorphism

$$
X \in \mathfrak{M}_{n}^{+} \rightarrow X^{\prime-1} \text {. }
$$

REMARK. When $n=2$, the automorphism (ii) is the same as $X \rightarrow S X S^{-1}$, hence is included in (i). The automorphism $X \rightarrow \epsilon(X) \cdot X$ occurs only for $n=2$. Furthermore, for odd $n$ all automorphisms of $\mathfrak{M}_{n_{j}}^{+}$are induced by automorphisms of $\mathfrak{M}_{n}$.

THEOREM 4. The generators of $\mathfrak{A}_{n}$ are

(i) the set of all inner automorphisms

$$
X \in \mathfrak{M}_{n} \rightarrow A X A^{-1}
$$


(ii) the automorphism $X \in \mathfrak{M}_{n} \rightarrow X^{\prime-1}$,

(iii) for even $n$ only, the automorphism

$$
X \in \mathfrak{M}_{n} \rightarrow(\operatorname{det} X) \cdot X,
$$

and

(iv) for $n=2$ only, the automorphism

$$
X \in \mathfrak{M}_{2}^{+} \rightarrow \epsilon(X) \cdot X, \quad X \in \mathfrak{M}_{2}^{-} \rightarrow \epsilon(J X) \cdot X,
$$

where $J$ is given by (2).

Further, when $n=2$, the automorphism (ii) may be omitted from this list.

Let us show that Theorem 4 is a simple consequence of Theorem 3. Let $\tau$ be any automorphism of $\mathfrak{M}_{n}$. By Corollary $1, \tau$ induces an automorphism on $\mathfrak{M}_{n}^{+}$which, by Theorems 2 and 3, can be written as:

$$
X \in \mathfrak{M}_{n}^{+} \rightarrow \alpha(X) \cdot A X^{*} A^{-1},
$$

where $A \in \mathfrak{M}_{n}, \alpha(X)=1$ for all $X$ or $\alpha(X)=\epsilon(X)$ for all $X$ (this can occur only when $n=2$ ), and where either $X^{*}=X$ for all $X$ or $X^{*}=X^{\prime-1}$ for all $X$.

Let $Y$ and $Z \in \mathfrak{M}_{n}^{-}$; then

$$
Y^{\tau} Z^{\tau}=(Y Z)^{\tau}=\alpha(Y Z) \cdot A(Y Z)^{*} A^{-1},
$$

whence

$$
Y^{\tau}=\alpha(Y Z) \cdot A Y^{*} Z^{*} A^{-1}\left(Z^{\tau}\right)^{-1} .
$$

Let $Z \in \mathfrak{M}_{n}^{-}$be fixed; then

$$
Y^{\tau}=\alpha(Y Z) \cdot A Y^{*} B \quad \text { for all } Y \in \mathfrak{M}_{n}^{-},
$$

where $A$ and $B$ are independent of $Y$. But then

$$
A Y^{*} B \cdot A Y^{*} B=\left(Y^{\tau}\right)^{2}=\left(Y^{2}\right)^{\tau}=\alpha\left(Y^{2}\right) A\left(Y^{2}\right)^{*} A^{-1},
$$

so that

$$
(B A) Y^{*}(B A)=\alpha\left(Y^{2}\right) Y^{*} .
$$

Since this is valid for all $Y \in \mathfrak{M}_{n}^{-}$, we see that of necessity $\alpha\left(Y^{2}\right)=1$ for all $Y$, and $B A= \pm I$. This shows that either $Y^{r}=\alpha(Y Z) \cdot A Y^{*} A^{-1}$ for all $Y \in \mathfrak{M}_{n}^{-}$, or $Y^{r}=-\alpha(Y Z) \cdot A Y^{*} A^{-1}$ for all $Y \in \mathfrak{M}_{n}^{-}$. If $n=2$ and $\alpha(Y Z)=\epsilon(Y Z)$, it is trivial to verify that either $\epsilon(Y Z)=\epsilon(J Y)$ for all $Y \in \mathfrak{M}_{2}^{-}$or $\epsilon(Y Z)=-\epsilon(J Y)$ for all $Y \in \mathfrak{M}_{2}^{-}$.

The remainder of the paper will be concerned with proving Theorem 3.

4. Canonical forms for involutions. In the proof of Theorem 3 we shall use certain canonical forms of involutions under similarity transformations.

LeMma 1. Under a similarity transformation, every involution $X \in \mathfrak{M}_{n}$ such 
that $X^{2}=I^{(n)}$ can be brought into the form

$$
W(x, y, z)=L+\underset{(x \text { terms })}{+}+L \dot{+}+(-I)^{(y)}+I^{(z)},
$$

where $2 x+y+z=n$ and

$$
L=\left(\begin{array}{rr}
1 & 0 \\
1 & -1
\end{array}\right)
$$

Proof. We prove first, by induction on $n$, that every $X \in \mathfrak{M}_{n}$ satisfying $X^{2}=I$ is similar to a matrix of the form

$$
\left(\begin{array}{cc}
I^{(l)} & 0 \\
M & -I^{(n-l)}
\end{array}\right) .
$$

For $n=1$ and 2, this is trivial. Let the theorem be proved for $n$, and assume that $X^{2}=I^{(n+1)}$, where $n \geqq 2$. Then $X^{2}-I=0$, or $(X-I)(X+I)=0$. If $X-I$ is nonsingular, then $X=-I$ and the result is obvious. Hence, supposing that $X-I$ is singular (so that $\lambda=1$ is a characteristic root of $X$ ), there exists a primitive column vector $\mathrm{t}=\left(t_{1}, \cdots, t_{n+1}\right)^{\prime}$ with integral elements such that $\mathbf{t}^{\prime} X=\mathbf{t}^{\prime}$. Choose $P \in \mathbb{M}_{n+1}$ with first row $\mathbf{t}^{\prime}$. Then

$$
P X P^{-1}=\left(\begin{array}{cc}
1 & \mathfrak{n}^{\prime} \\
\mathfrak{x} & X_{1}
\end{array}\right),
$$

where $\mathfrak{n}$ denotes a vector whose components are 0 ; thus

$$
X \stackrel{s}{=}\left(\begin{array}{ll}
1 & \mathfrak{n}^{\prime} \\
\mathfrak{x} & X_{1}
\end{array}\right) .
$$

But

$$
I^{(n+1)}=X^{2} \stackrel{\bullet}{=}\left(\begin{array}{cc}
1 & \mathfrak{n}^{\prime} \\
\left(I+X_{1}\right) \mathfrak{x} & X_{1}^{2}
\end{array}\right)
$$

shows that $X_{1}^{2}=I^{(n)}$ and $\left(I+X_{1}\right) \mathfrak{x}=\mathfrak{n}$. By the induction hypothesis,

$$
X_{1} \stackrel{\bullet}{=}\left(\begin{array}{lc}
I^{(m)} & 0 \\
M & -I^{(n-m)}
\end{array}\right),
$$

and, after making the similarity transformation, we have (as a consequence of $\left.\left(I+X_{1}\right) \mathfrak{x}=\mathfrak{n}\right)$

$$
\left(\begin{array}{ll}
2 I^{(m)} & 0 \\
\mathbb{Z} M & 0
\end{array}\right) \mathfrak{x}=\mathfrak{n} .
$$

Therefore 


$$
\mathfrak{x}=\underset{(m \text { terms })}{(0, \cdots,} \underset{(n-m \text { terms })}{*}, \cdots,
$$

where $*$ denotes an arbitrary element. Thus

$$
X \stackrel{s}{=}\left(\begin{array}{cccc}
1 & & \mathfrak{n}^{\prime} & \\
0 & & & \\
\vdots & I^{(m)} & 0 \\
0 & & \\
* & & -I^{(n-m)} \\
\cdot & M &
\end{array}\right)=\left(\begin{array}{cc}
I^{(m+1)} & 0 \\
\bar{M} & -I^{(n-m)}
\end{array}\right)
$$

This completes the first part of the proof.

Suppose we now subject (5) to a further similarity transformation by

$$
\left(\begin{array}{cc}
A^{(l)} & 0 \\
C & D^{(n-l)}
\end{array}\right) \in \mathfrak{M}_{n} .
$$

A simple calculation shows that we obtain a matrix given by (5) with $M$ replaced by $\bar{M}$, where $\bar{M}=2 C A^{-1}+D M A^{-1}$. Choosing firstly $C=0, A$ and $D$ unimodular, we find that $\bar{M}=D M A^{-1}$, and by proper choice of $A$ and $D$ we can make $\bar{M}$ diagonal. Supposing this done, secondly put $A=I, D=I$; we find that $\bar{M}=M+2 C$. Since $C$ is arbitrary, we can bring $\bar{M}$ into the form

$$
\left(\begin{array}{ll}
I^{(k)} & 0 \\
0 & 0
\end{array}\right)
$$

where $k$ is the rank of $M$. Since we can interchange two rows and simultaneously interchange the corresponding columns by means of a similarity transformation, the lemma follows.

It is easily seen that

$$
W(x, y, z) \stackrel{\bullet}{=} W(\bar{x}, \bar{y}, \bar{z})
$$

only when $x=\bar{x}, y=\bar{y}$, and $z=\bar{z}$. Furthermore, changing the order of terms in the direct summation does not alter the similarity class. The number $A_{n}$ of nonsimilar involutions in $\mathfrak{M}_{n}$ is therefore equal to the number of solutions of $2 x+y+z=n, x \geqq 0, y \geqq 0, z \geqq 0$. This gives

$$
A_{n}= \begin{cases}\left(\frac{n+2}{2}\right)^{2}, & n \text { even } \\ \frac{(n+1)(n+3)}{4}, & n \text { odd }\end{cases}
$$


Let $B_{n}$ be the number of nonsimilar involutions in $\mathfrak{M}_{n}^{+}$, where the similarity factors are in $\mathfrak{M}_{n}$. One easily obtains

$$
B_{n}=\left\{\begin{array}{lr}
\left(A_{n}-1\right) / 2, & \text { if } n \equiv 0(\bmod 4), \\
A_{n} / 2, & \text { otherwise. }
\end{array}\right.
$$

5. Automorphisms of $\mathfrak{M}_{3}^{+}$. We shall now prove Theorem 3 for $n=3$. Let

$$
I_{1}=\left(\begin{array}{rrr}
-1 & 0 & 0 \\
0 & -1 & 0 \\
0 & 0 & 1
\end{array}\right), \quad I_{2}=\left(\begin{array}{rrr}
1 & 0 & 0 \\
1 & -1 & 0 \\
0 & 0 & -1
\end{array}\right) \in \mathfrak{M}_{3}^{+} .
$$

Then $I_{1}^{2}=I^{(3)}$. Let $\tau$ be any automorphism of $\mathfrak{M}_{3}^{+}$and let $X=I_{1}^{\tau}$; then $X^{2}=I^{(3)}$. By Lemma 1, the matrices $I_{1}, I_{2}$, and $I^{(3)}$ form a complete system of nonsimilar involutions in $\mathfrak{M}_{3}^{+}$. Therefore

$$
X \stackrel{8}{=} I_{1} \text { or } I_{2} \text {. }
$$

After a suitable inner automorphism, we may assume that either $I_{1} \rightarrow I_{1}$ or $I_{1} \rightarrow I_{2}$. We shall show that this latter case is impossible by considering the normalizer groups of $I_{1}$ and $I_{2}$. The normalizer group of $I_{1}$, that is, the group of matrices $\in \mathfrak{M}_{3}^{+}$which commute with $I_{1}$, consists of all elements of $\mathfrak{M}_{3}^{+}$of the form

$$
\left(\begin{array}{lll}
a & b & 0 \\
c & d & 0 \\
0 & 0 & e
\end{array}\right)
$$

and is isomorphic to $\mathfrak{M}_{2}$. That of $I_{2}$ consists of all elements of $\mathfrak{M}_{3}^{+}$of the form

$$
\left(\begin{array}{ccc}
a & 0 & 0 \\
(a-e) / 2 & e & f \\
-h / 2 & h & i
\end{array}\right)
$$

and is isomorphic to that subgroup $\mathbb{B}$ of $\mathfrak{M}_{2}$ consisting of the elements

$$
\left.\left(\begin{array}{rr}
e & f \\
h & i
\end{array}\right) \in \mathfrak{M}_{2}, \quad \begin{array}{rl}
e & \equiv 1 \\
h & \equiv 0 \\
i & \equiv 1
\end{array}\right\}(\bmod 2) .
$$

Since $e$ and $i$ are both odd, $\$ 3$ contains no element of order 3 , and hence is not isomorphic to $\mathfrak{M}_{2}$. But then $I_{1} \rightarrow I_{2}$ is impossible.

We may assume thus that after a suitable inner automorphism, $I_{1}$ is invariant. Thence elements of $\mathfrak{M}_{3}^{+}$which commute with $I_{1}$ map into elements of the same kind, so that 


$$
\left(\begin{array}{cc}
X & \mathfrak{n}^{\prime} \\
\mathfrak{n} & \pm 1
\end{array}\right) \in \mathfrak{M}_{3}^{+} \rightarrow\left(\begin{array}{cc}
X^{r} & \mathfrak{n}^{\prime} \\
\mathfrak{n} & \pm 1
\end{array}\right)
$$

Since this induces an automorphism $X \rightarrow X^{\tau}$ on $\mathfrak{M}_{2}$, we see that $\operatorname{det} X^{\tau}=\operatorname{det} X$, and hence the plus signs go together, and so do the minus signs. By Theorem 2 and that part of Theorem 4 which follows from Theorem 2, there exists a matrix $A \in \mathfrak{M}_{2}$ such that $X^{r}= \pm A X A^{-1}$; here, the plus sign certainly occurs when $X$ is an even element of $\mathfrak{M}_{2}^{+}$, and if the minus sign occurs for one odd element of $\mathfrak{M}_{2}^{+}$, then it occurs for every odd element of $\mathfrak{M}_{2}^{+}$. By use of a further inner automorphism using the factor $A^{-1} \dot{+} I^{(1)}$, we may assume that

$$
\left(\begin{array}{cc}
X & \mathfrak{n}^{\prime} \\
\mathfrak{n} & \pm 1
\end{array}\right) \in \mathfrak{M}_{3}^{+} \rightarrow\left(\begin{array}{cc} 
\pm X & \mathfrak{n}^{\prime} \\
\mathfrak{n} & \pm 1
\end{array}\right)
$$

so that

$$
M=\left(\begin{array}{rrr}
1 & 0 & 0 \\
0 & -1 & 0 \\
0 & 0 & -1
\end{array}\right) \rightarrow M \quad \text { or } \quad M \rightarrow N=\left(\begin{array}{rrr}
-1 & 0 & 0 \\
0 & 1 & 0 \\
0 & 0 & -1
\end{array}\right)
$$

Since

$$
N=\left(\begin{array}{rrr}
0 & -1 & 0 \\
1 & 0 & 0 \\
0 & 0 & 1
\end{array}\right) \cdot M \cdot\left(\begin{array}{rrr}
0 & 1 & 0 \\
-1 & 0 & 0 \\
0 & 0 & 1
\end{array}\right)
$$

we may assume (after a further inner automorphism, if necessary) that $I_{1}$, $M$, and $N$ are all invariant under the automorphism (but (8) need not hold).

Thus, after a suitably chosen inner automorphism, we have $I_{1}, M$, and $N$ invariant. Therefore there exist $A, B$, and $C \in \mathfrak{M}_{2}$ such that

$$
\begin{aligned}
\left(\begin{array}{rr}
X & \mathfrak{n} \\
\mathfrak{n}^{\prime} & \pm 1
\end{array}\right) & \in \mathfrak{M}_{3}^{+} \rightarrow\left(\begin{array}{rr} 
\pm A X A^{-1} & \mathfrak{n} \\
\mathfrak{n}^{\prime} & \pm 1
\end{array}\right), \\
\left(\begin{array}{rr} 
\pm 1 & \mathfrak{n}^{\prime} \\
\mathfrak{n} & X
\end{array}\right) & \in \mathfrak{M}_{3}^{+} \rightarrow\left(\begin{array}{rc} 
\pm 1 & \mathfrak{n}^{\prime} \\
\mathfrak{n} & \pm B X B^{-1}
\end{array}\right), \\
\left(\begin{array}{rrr}
a & 0 & b \\
0 & \pm 1 & 0 \\
c & 0 & d
\end{array}\right) & \in \mathfrak{M}_{3}^{+} \rightarrow\left(\begin{array}{rrr}
\alpha & 0 & \beta \\
0 & \pm 1 & 0 \\
\gamma & 0 & \delta
\end{array}\right),
\end{aligned}
$$

where

$$
\left(\begin{array}{ll}
\alpha & \beta \\
\gamma & \delta
\end{array}\right)= \pm C\left(\begin{array}{ll}
a & b \\
c & d
\end{array}\right) C^{-1}
$$

and $\mathfrak{n}=(0,0)^{\prime}$. Here, the +1 on the left goes with the +1 on the right al- 
ways (and the -1 's go together); further, when $X$ is an even element of $\mathfrak{M}_{2}^{+}$, the plus sign occurs before $A X A^{-1}, B X B^{-1}$, and $C X C^{-1}$, while if the minus sign occurs before one of these for any odd $X \in \mathfrak{M}_{2}^{+}$, it occurs there for every odd $X \in \mathfrak{M}_{2}^{+}$.

Now we may assume that at most one of $A, B$, and $C$ has determinant -1; for if both $A$ and $B$ (say) have determinant -1 , apply a further inner automorphism (with factor $N$ ) which leaves $I_{1}, M$, and $N$ invariant and changes the signs of $\operatorname{det} A$ and $\operatorname{det} B$. Suppose hereafter, without loss of generality, that $\operatorname{det} A=\operatorname{det} B=1$.

Next, $N$ is invariant, but by (9) goes into

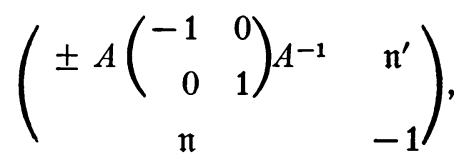

so that

$$
\pm A\left(\begin{array}{rr}
-1 & 0 \\
0 & 1
\end{array}\right) A^{-1}=\left(\begin{array}{rr}
-1 & 0 \\
0 & 1
\end{array}\right)
$$

This gives two possibilities:

$$
A=I^{(2)} \text { or }\left(\begin{array}{rr}
0 & 1 \\
-1 & 0
\end{array}\right)
$$

The same holds true for $B$ (but not necessarily for $C$, since $\operatorname{det} C= \pm 1$ ).

Suppose firstly that either $A$ or $B$ is $I^{(2)}$, say $A=I^{(2)}$. Then

$$
T=\left(\begin{array}{lll}
1 & 1 & 0 \\
0 & 1 & 0 \\
0 & 0 & 1
\end{array}\right) \rightarrow\left(\begin{array}{ccc} 
\pm\left(\begin{array}{ll}
1 & 1 \\
0 & 1
\end{array}\right) & 0 \\
0 & 0 & 1
\end{array}\right)
$$

Case 1. $T$ invariant. Then

$$
\left(\begin{array}{rrr}
0 & 1 & 0 \\
-1 & 0 & 0 \\
0 & 0 & 1
\end{array}\right) \text { and }\left(\begin{array}{rrr}
0 & 1 & 0 \\
1 & 0 & 0 \\
0 & 0 & -1
\end{array}\right)
$$

are both invariant. (The first matrix is invariant in virtue of the remarks after (9); the second is invariant because it is $M$ times the first.) For either possible choice of $B$ we find that

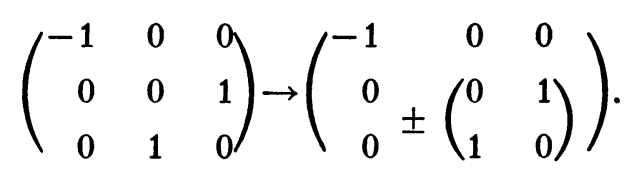


Therefore

$$
U=\left(\begin{array}{lll}
0 & 1 & 0 \\
0 & 0 & 1 \\
1 & 0 & 0
\end{array}\right)=\left(\begin{array}{rrr}
-1 & 0 & 0 \\
0 & -1 & 0 \\
0 & 0 & 1
\end{array}\right)\left(\begin{array}{rrr}
-1 & 0 & 0 \\
0 & 0 & 1 \\
0 & 1 & 0
\end{array}\right)\left(\begin{array}{rrr}
0 & 1 & 0 \\
1 & 0 & 0 \\
0 & 0 & -1
\end{array}\right)
$$

is mapped into

$$
\left(\begin{array}{rrr}
-1 & 0 & 0 \\
0 & -1 & 0 \\
0 & 0 & 1
\end{array}\right)\left(\begin{array}{rrr}
-1 & 0 & 0 \\
0 \\
0
\end{array} \pm\left(\begin{array}{lll}
0 & 1 \\
1 & 0
\end{array}\right)\right)\left(\begin{array}{rrr}
0 & 1 & 0 \\
1 & 0 & 0 \\
0 & 0 & -1
\end{array}\right)=\left\{\begin{array}{l}
U, \text { if }+ \text { is used } \\
V, \text { if }- \text { is used }
\end{array}\right.
$$

where $V=I_{1} U I_{1}^{-1}$. Thus, in this case, $T \rightarrow T=I_{1} T I_{1}^{-1}$, and either $U \rightarrow U$ or $U \rightarrow I_{1} U I_{1}^{-1}$. Since $T$ and $U$ generate $\left({ }^{2}\right) \mathfrak{M}_{3}^{+}$, the automorphism is inner.

Case 2.

$$
T \rightarrow\left(\begin{array}{rrr}
-1 & -1 & 0 \\
0 & -1 & 0 \\
0 & 0 & 1
\end{array}\right)
$$

Then

$$
\left(\begin{array}{rrr}
0 & 1 & 0 \\
1 & 0 & 0 \\
0 & 0 & -1
\end{array}\right) \rightarrow\left(\begin{array}{rrr}
0 & -1 & 0 \\
-1 & 0 & 0 \\
0 & 0 & -1
\end{array}\right)
$$

and one finds in this case that

$$
U \rightarrow\left(\begin{array}{rrr}
0 & -1 & 0 \\
0 & 0 & 1 \\
-1 & 0 & 0
\end{array}\right) \text { or }\left(\begin{array}{rrr}
0 & -1 & 0 \\
0 & 0 & -1 \\
1 & 0 & 0
\end{array}\right)
$$

If we set $Z=T U^{2}$, then

$$
\left(\begin{array}{lll}
1 & 0 & 0 \\
1 & 1 & 0 \\
0 & 0 & 1
\end{array}\right)=\left(U Z^{-1}\right)^{2} U Z^{2}
$$

Now certainly the left side of (10) maps into

$$
\left(\begin{array}{rrr}
-1 & 0 & 0 \\
-1 & -1 & 0 \\
0 & 0 & 1
\end{array}\right)
$$

(2) L. K. Hua and I. Reiner, loc. cit. 
whereas, knowing $T^{r}$ and $U^{r}$, we can compute $Z^{r}$ and thence can find the image of the right side of (10). We readily find (for either value of $U^{r}$ ) that the right side of (10) maps into

$$
\left(\begin{array}{lll}
1 & \cdot & \cdot \\
3 & \cdot & \cdot \\
. & \cdot & .
\end{array}\right)
$$

and hence we have a contradiction.

Therefore case 2 cannot occur, and so if either $A$ or $B$ equals $I^{(2)}$, the automorphism is inner. Suppose hereafter that

$$
A=B=\left(\begin{array}{rr}
0 & 1 \\
-1 & 0
\end{array}\right)
$$

In this case we have

$$
T \rightarrow\left(\begin{array}{rrr} 
\pm\left(\begin{array}{rr}
1 & 0 \\
-1 & 1
\end{array}\right) & 0 \\
0 & 0 & 1
\end{array}\right)
$$

Case 1*.

$$
T \rightarrow\left(\begin{array}{rrr}
1 & 0 & 0 \\
-1 & 1 & 0 \\
0 & 0 & 1
\end{array}\right)
$$

Then as before

$$
\left(\begin{array}{rrr}
0 & 1 & 0 \\
-1 & 0 & 0 \\
0 & 0 & 1
\end{array}\right) \text { and }\left(\begin{array}{rrr}
0 & 1 & 0 \\
1 & 0 & 0 \\
0 & 0 & -1
\end{array}\right)
$$

are invariant, and again $U^{r}=U$ or $V$. After a further inner automorphism by a factor of $I_{1}$ (in the latter case) we also have $U \rightarrow U$. But then

$$
T \rightarrow T^{\prime-1}, \quad U \rightarrow U^{\prime-1} .
$$

(This automorphism is easily shown to be a non-inner automorphism.)

Case 2*.

$$
T \rightarrow\left(\begin{array}{rrr}
-1 & 0 & 0 \\
1 & -1 & 0 \\
0 & 0 & z_{1}
\end{array}\right)
$$

Then 


$$
\left(\begin{array}{rrr}
0 & 1 & 0 \\
1 & 0 & 0 \\
0 & 0 & -1
\end{array}\right) \rightarrow\left(\begin{array}{rrr}
0 & -1 & 0 \\
-1 & 0 & 0 \\
0 & 0 & -1
\end{array}\right)
$$

and again we find that there are two possibilities for $U^{r}$, each of which leads to a contradiction, just as in case 2. Therefore Theorem 3 holds when $n=3$.

6. A fundamental lemma. Theorem 3 will be proved by induction on $n$; the result has already been established for $n=2$ and 3 . In going from $n-1$ to $n$, the following lemma is basic:

LeMma 2. Let $n \geqq 4$, and define $J_{1}=(-1)+I^{(n-1)}$. In any automorphism $\tau$ of $\mathfrak{M}_{n}, J_{1}^{r}= \pm A J_{1} A^{-1}$ for some $A \in \mathfrak{M}_{n}$.

Proof. By Corollary 1, $J_{1}^{\tau} \in \mathfrak{M}_{n}^{-}$, and $J_{1}^{\tau}$ is an involution. After a suitable inner automorphism, we may assume that $J_{1}^{r}=W(x, y, z)$ (as defined by (4)), where $2 x+y+z=n$ and $x+y$ is odd. Every element of $\mathfrak{M}_{n}$ which commutes with $J_{1}$ maps into an element of $\mathfrak{M}_{n}$ which commutes with $W$. Every matrix in $\mathfrak{M}_{n}^{+}$maps into a matrix in $\mathfrak{M}_{n}^{+}$. Combining these facts, we see that the group $\mathfrak{S}_{1}$ consisting of those elements of $\mathfrak{M}_{n}^{+}$which commute with $J_{1}$ is isomorphic to $\mathbb{S}_{2}$, the corresponding group for $W$. If we prove that this can happen only for $x=0, y=1, z=n-1$ or $x=0, y=n-1, z=1$, the result will follow.

The group $\mathfrak{S}_{1}$ consists of the matrices in $\mathfrak{M}_{n}^{+}$of the form $( \pm 1) \dot{+} X_{1}$, $X_{1} \in \mathfrak{M}_{n-1}$, and so clearly $\mathfrak{S}_{1} \cong \mathfrak{M}_{n-1}$.

The group $\mathfrak{H}_{2}$ is easily found to consist of all matrices $C \in \mathfrak{M}_{1}^{+}$of the form (we illustrate the case where $x=2$ ):

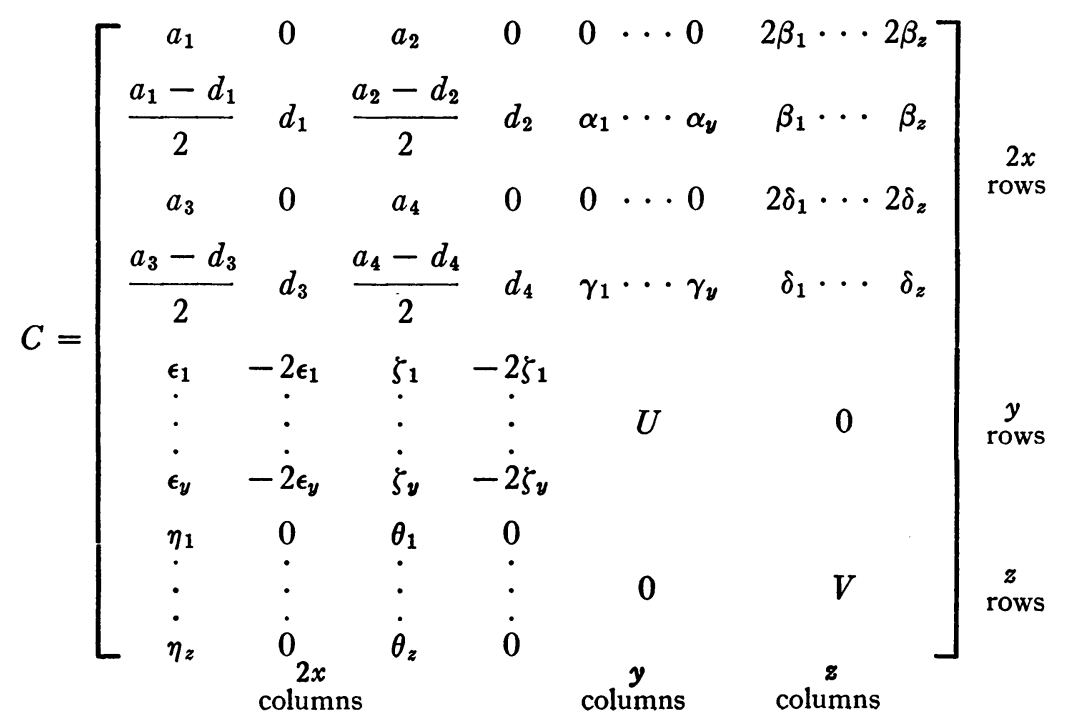


For the moment put

$$
K=\left(\begin{array}{cc}
1 & 0 \\
-1 / 2 & 1
\end{array}\right)+\underset{(x \text { terms })}{\ldots}+\left(\begin{array}{cc}
1 & 0 \\
-1 / 2 & 1
\end{array}\right)+I^{(n-2 x)} .
$$

Then a simple calculation gives:

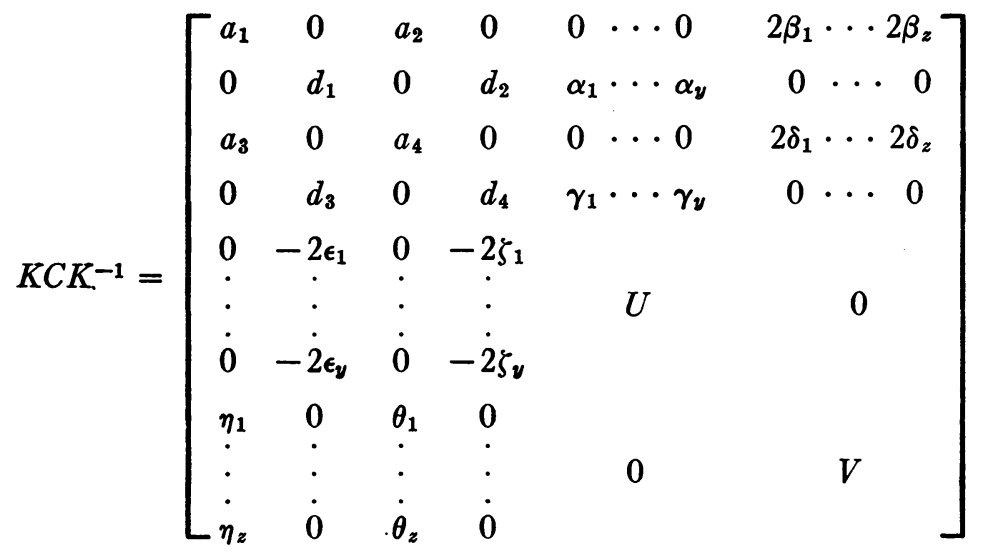

and so $C$ is similar to

$$
\begin{aligned}
& {\left[\begin{array}{ccc}
a_{1} & a_{2} & 2 \beta_{1} \cdots 2 \beta_{z} \\
a_{3} & a_{4} & 2 \delta_{1} \cdots 2 \delta_{z} \\
\eta_{1} & \theta_{1} & \\
\vdots & \vdots & V \\
\eta_{z} & \dot{\theta}_{z} &
\end{array}\right]+\left[\begin{array}{ccc}
d_{1} & d_{2} & \alpha_{1} \cdots \alpha_{y} \\
d_{3} & d_{4} & \gamma_{1} \cdots \gamma_{y} \\
-2 \epsilon_{1} & -2 \zeta_{1} & \\
\vdots & \vdots & U \\
-2 \epsilon_{y} & -2 \zeta_{y} &
\end{array}\right]}
\end{aligned}
$$

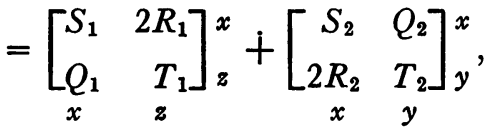

with a fixed similarity factor depending only on $W$. Therefore $\mathbb{S}_{2} \cong(5$, where $\mathbb{B}=\mathbb{S}(x, y, z)$ is the group of matrices in $\mathfrak{M}_{n}^{+}$of the form

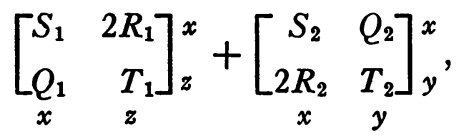

where $S_{1} \equiv S_{2}(\bmod 2)$. Here $2 x+y+z=n$ and $x+y$ is odd.

We wish to prove that $\mathfrak{M}_{n-1} \cong \mathfrak{S}(x, y, z)$ only when $x=0, y=1, z=n-1$ or $x=0, y=n-1, z=1$. In order to establish this, we shall prove that in all other cases the number of involutions in $\$$ which are nonsimilar in $\$$ is greater than the number of involutions in $\mathfrak{M}_{n-1}$ which are nonsimilar in $\mathfrak{M}_{n-1}$; 
this latter number is, of course, $A_{n-1}$ (given by (6)).

We shall briefly denote the elements of (S) by $A+B$, where

$$
A=\left(\begin{array}{cc}
S_{1} & 2 R_{1} \\
Q_{1} & T_{1}
\end{array}\right) \text { and } B=\left(\begin{array}{cc}
S_{2} & Q_{2} \\
2 R_{2} & T_{2}
\end{array}\right)
$$

If $A_{1}+B_{1}$ and $A_{2}+B_{2}$ are two involutions in $\$(S)$ where either

$$
A_{1} \stackrel{8}{\neq} A_{2}
$$

in $\mathfrak{M}_{x+z}$ or

$$
B_{1} \not B_{2}
$$

in $\mathfrak{M}_{x+y}$, then certainly

$$
A_{1} \dot{+} B_{1} \stackrel{\bullet}{\neq} A_{2}+B_{2}
$$

in $\mathfrak{S}$ (these may be similar in $\mathfrak{M}_{n}$, however). Therefore, the matrices $A \dot{+} B$, where

$$
\begin{aligned}
& A=I^{\left(a_{1}\right)}+(-I)^{\left(b_{1}\right)}+L+\underset{\left(c_{1} \text { terms }\right)}{\ldots}+L, \\
& B=I^{\left(a_{2}\right)}+(-I)^{\left(b_{2}\right)}+L+\underset{\left(c_{2} \text { terms }\right)}{+}+L,
\end{aligned}
$$

obtained by taking different sets of values of $\left(a_{1}, b_{1}, c_{1}, a_{2}, b_{2}, c_{2}\right)$, if they lie in $\$$, are certainly nonsimilar in $\$$. Here we have

$$
a_{1}+b_{1}+2 c_{1}=x+z, \quad a_{2}+b_{2}+2 c_{2}=x+y, \quad b_{1}+b_{2}+c_{1}+c_{2} \text { even. }
$$

If $x \neq 0$, we impose the further restriction that $c_{1} \leqq(z+1) / 2, c_{2} \leqq(y+1) / 2$, and that in $B$ instead of $L$ we use $L^{\prime}$. These conditions will insure that $A+B \in(3)$. We certainly do not (in general) get all of the nonsimilar involutions of $B$ in this way, but instead we obtain only a subset thereof. Call the number of such matrices $N$.

For $x=0$, we have $N=B_{y} B_{z}+\left(A_{y}-B_{y}\right)\left(A_{z}-B_{z}\right)$. Since $y$ is odd, $A_{y}=2 B_{y}$, and therefore

$$
N=B_{y} A_{z}=B_{y} A_{n-y} .
$$

Case 1. $n$ even. Then $N=(y+1)(y+3)(n-y+1)(n-y+3) / 32$. If neither $y$ nor $n-y$ is 1 (certainly neither can be zero), then

$$
(y+1)(n-y+1) \geqq 4(n-2) \text { and }(y+3)(n-y+3) \geqq 6 n,
$$

so that

$$
N \geqq(24 / 32) n(n-2) .
$$

For $n=4, x=0$, either $y=1$ or $z=1$. For $n \geqq 6$, we have $N>A_{n-1}$. Hence in 
this case (S) is not isomorphic to $\mathfrak{M}_{n-1}$. (If either $y$ or $n-y=1$, then $W(x, y, z)$ $= \pm J_{1}$.)

Case 2. $n$ odd. Then $N=(y+1)(y+3)(n-y+2)^{2} / 32$. We find again that $N>A_{n-1}$ for $n \geqq 5$.

This settles the cases where $x=0$. Suppose that $x \neq 0$ hereafter. Then $N$ is the number of solutions of

$$
\begin{gathered}
a_{1}+b_{1}+2 c_{1}=x+z, a_{2}+b_{2}+2 c_{2}=x+y, \quad b_{1}+b_{2}+c_{1}+c_{2} \text { even, } \\
0 \leqq c_{1} \leqq \frac{z+1}{2}, \quad 0 \leqq c_{2} \leqq \frac{y+1}{2} .
\end{gathered}
$$

Using $[r]$ to denote the greatest integer less than or equal to $r$, we readily find that $N$ is given by

$$
\frac{1}{2}\left[\frac{z+3}{2}\right]\left[\frac{y+3}{2}\right]\left(x+z+1-\left[\frac{z+1}{2}\right]\right)\left(x+y+1-\left[\frac{y+1}{2}\right]\right) .
$$

By considering separately the cases where $y$ and $z$ are both even, one even and one odd, and so on, it is easy to prove that $N \geqq A_{n-1}$ in all cases except when both $y$ and $z$ are zero. Leaving aside this case for the moment, consider the matrix $A_{0} \dot{+} I^{(x+y)} \in\left(\mathfrak{S}\right.$, where $A_{0} \in \mathfrak{M}_{x+z}$ is given by

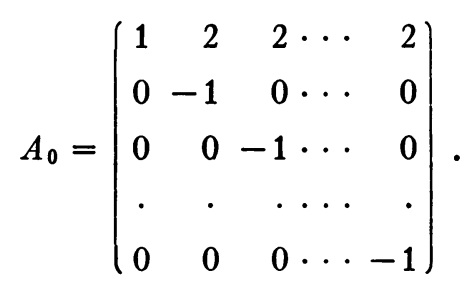

The matrix $A_{0} \dot{+} I^{(x+y)}$ is certainly an involution in $\mathcal{G}$. Since, in $\mathfrak{M}_{x+z}$,

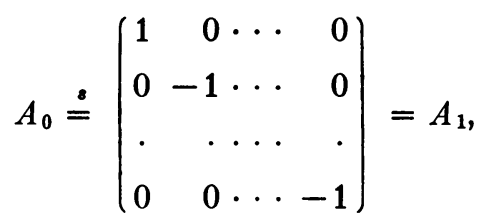

$A_{0}+I^{(x+y)}$ can be similar (in (S) only to that matrix (counted in the $N$ matrices) of the form $A_{1}+I^{(x+y)}$. But from

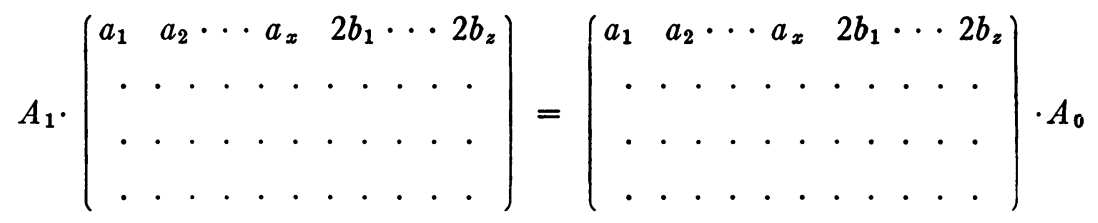

we obtain 


$$
a_{1}=a_{2}=\cdots=a_{x}=2 b_{1},
$$

which is impossible. Hence (s) contains at least $N+1$ nonsimilar involutions, and therefore $(5)$ is not isomorphic to $\mathfrak{M}_{n-1}$ in these cases.

We have left only the case $y=z=0, x=n / 2$; then $n$ is singly even. Here we may choose $A=W\left(c_{1}, b_{1}, a_{1}\right), B=W\left(c_{1}, b_{2}, a_{2}\right)$, where

$$
a_{1}+b_{1}+2 c_{1}=x, \quad a_{2}+b_{2}+2 c_{1}=x, \quad b_{1}+b_{2} \text { even. }
$$

Then $A+B \in(5$, and the various matrices are nonsimilar. The number of such matrices is $(x+1)(x+2)(x+3) / 12$, which is greater than $A_{n-1}$ for $n \geqq 14$. For $n=6, \mathfrak{M}_{n-1}$ contains an element of order 5 , while $\&$ does not. For $n=10$, $\mathfrak{M}_{n-1}$ contains an element of order 7 , while $(5)$ does not. This completes the proof of the lemma.

7. Proof of Theorem 3. We are now ready to give a proof of Theorem 3 by induction on $n$. Hereafter, let $n \geqq 4$ and suppose that Theorem 3 holds for $n-1$. If $\tau$ is any automorphism of $\mathfrak{M}_{n}$, by Corollary 1 and Lemma 2 we know that $\tau$ takes $\mathfrak{M}_{n}^{+}$into itself, and $J_{1}^{\tau}= \pm A J_{1} A^{-1}$. If we change $\tau$ by a suitable inner automorphism, then we may assume that $J_{1} \rightarrow \pm J_{1}$. When $n$ is odd, certainly $J_{1} \rightarrow J_{1}$; when $n$ is even, by multiplying $\tau$ by the automorphism $X \in \mathfrak{M}_{n} \rightarrow(\operatorname{det} X) \cdot X$ if necessary, we may again assume $J_{1} \rightarrow J_{1}$.

Therefore, every $M_{\infty} \in \mathfrak{M}_{n}^{+}$which commutes with $J_{1}$ goes into another such element, that is,

$$
\left(\begin{array}{rr} 
\pm 1 & \mathfrak{n}^{\prime} \\
\mathfrak{n} & X
\end{array}\right)^{\tau}=\left(\begin{array}{rr} 
\pm 1 & \mathfrak{n}^{\prime} \\
\mathfrak{n} & X^{\tau}
\end{array}\right)
$$

Since this induces an automorphism on $\mathfrak{M}_{n-1}$, we have $\operatorname{det} X^{\tau}=\operatorname{det} X$, so that the plus signs go together, as do the minus signs. Furthermore, by our induction hypothesis,

$$
X^{\tau}= \pm A X^{*} A^{-1}
$$

where $A \in \mathfrak{M}_{n-1}$ and either $X^{*}=X$ for all $X \in \mathfrak{M}_{n-1}$ or $X^{*}=X^{\prime-1}$ for all $X \in \mathfrak{M}_{n-1}$; here the minus sign can occur only for $X \in \mathfrak{M}_{n-1}^{-}$, and if it occurs for one such $X$, it occurs for all $X \in \mathfrak{M}_{n-1}^{-}$. After changing our original automorphism by a factor of $I^{(1)} \dot{+} A^{-1}$, we may assume that $X^{\tau}= \pm X^{*}$. Then

Let $J_{\nu}$ be obtained from $I^{(n)}$ by replacing the $\nu$ th diagonal element by -1 .

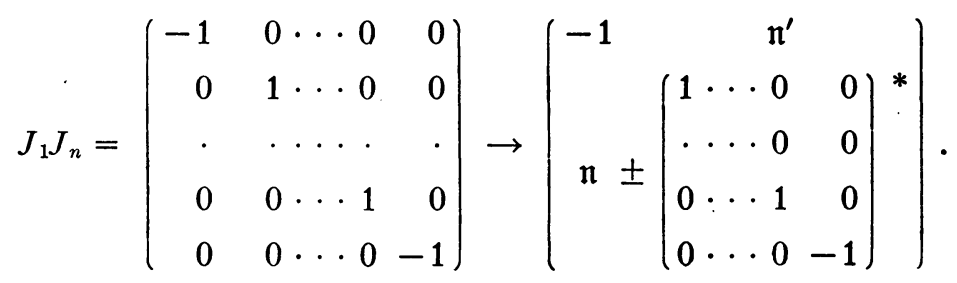


The minus sign here is impossible by Lemma 2 , since $n \geqq 4$. Hence $J_{1} J_{n}$ is invariant, and therefore so is $J_{n}$. By the same reasoning all of the $J_{\nu}(\nu=1, \cdots, n)$ are invariant.

From the above remarks we see that for $X \in \mathfrak{M}_{n-1}^{+}$,

$$
\left(\begin{array}{ll}
1 & \mathfrak{n}^{\prime} \\
\mathfrak{n} & X
\end{array}\right)^{\tau}=\left(\begin{array}{cc}
1 & \mathfrak{n}^{\prime} \\
\mathfrak{n} & A_{1} X^{*} A_{1}^{-1}
\end{array}\right), \ldots,\left(\begin{array}{ll}
X & \mathfrak{n} \\
\mathfrak{n}^{\prime} & 1
\end{array}\right)^{\tau}=\left(\begin{array}{cc}
A_{\mathfrak{n}} X^{*} A_{n}^{-1} & \mathfrak{n} \\
\mathfrak{n}^{\prime} & 1
\end{array}\right),
$$

where $A_{\nu} \in \mathfrak{M}_{n-1}$, and in fact $A_{1}=I$. Now suppose that $Z \in \mathfrak{M}_{n-2}^{+}$, and form $I^{(2)} \dot{+} Z$. Since it commutes with both $J_{1}$ and $J_{2}$, its image must do likewise. But then

$$
A_{1}\left(\begin{array}{ll}
1 & \mathfrak{n}^{\prime} \\
\mathfrak{n} & Z
\end{array}\right) A_{1}^{-1}=\left(\begin{array}{ll}
1 & \mathfrak{n}^{\prime} \\
\mathfrak{n} & \bar{Z}
\end{array}\right)
$$

for every $Z \in \mathfrak{M}_{n-2}^{+}$. Setting

$$
A_{1}=\left(\begin{array}{ll}
a & \mathfrak{x}^{\prime} \\
\mathfrak{y} & A
\end{array}\right)
$$

we obtain $\mathfrak{x}^{\prime} Z=\mathfrak{r}^{\prime}, \mathfrak{y}=\bar{Z} \mathfrak{y}$. Since this holds for all $Z \in \mathfrak{M}_{n-2}^{+}$, we must have $\mathfrak{x}=\mathfrak{y}=\mathfrak{n}$, so that $A_{1}$ is itself decomposable. A similar argument (considering the matrices commuting with both $J_{1}$ and $J_{\nu}$, for $\nu=3, \cdots, n$ ) shows that $A_{1}$ is diagonal. Correspondingly, all of the $A_{\nu}$ are diagonal. It is further clear that all of the $A_{\nu}(\nu=1, \cdots, n)$ are sections of a single diagonal matrix $D^{(n)}$. Using the further inner automorphism factor $D^{-1}$, we may henceforth assume that $X^{\tau}=X^{*}$ for every decomposable $X \in \mathfrak{M}_{n}^{+}$, where either $X^{*}=X$ always or $X^{*}=X^{\prime-1}$ always. Since $\mathfrak{M}_{n}^{+}$is generated by the set of decomposable elements of $\mathfrak{M}_{n}^{+}$, the theorem is proved.

Tsing Hua University, Peking, China.

UNIVERSITY OF ILLINOIS, URBANA, ILL. 